\title{
Memory confusions for real and imagined completions of symmetrical visual patterns
}

\author{
RONALD A. FINKE \\ State University of New York at Stony Brook, Stony Brook, New York \\ MARCIA K. JOHNSON \\ Princeton University, Princeton, New Jersey \\ and \\ GARY C. -W. SHYI \\ State University of New York at Stony Brook, Stony Brook, New York
}

\begin{abstract}
In Experiment 1, subject ratings indicated that it was easier to imaginally complete half of a symmetrical form when the form was symmetrical about the vertical axis than when the form was rotated so that it was symmetrical about the horizontal axis. In Experiment 2, new subjects saw these same forms, some presented as wholes and some presented as halves. Compared with controls, subjects who were instructed to imagine the vertically symmetrical half forms as complete had more difficulty remembering which forms had been presented as wholes and which had been presented as halves. Imagery and control groups did not differ in ability to discriminate half from whole horizontally symmetrical forms. The results are consistent with predictions from Johnson and Raye's (1981) reality monitoring model that discriminating memories of imagined and perceived events should be a function of the amount of cognitive operations included in the memory.
\end{abstract}

One of the basic issues in the study of human memory is how we are able to distinguish memories for events that were actually perceived from memories for events that were merely thought about or imagined. Our ability to do so is usually quite good, but it is far from perfect, and often we experience confusions in making these discriminations. For example, you might not remember whether you actually saw a set of keys in a particular place or only thought about seeing them. These types of memory confusions reveal failures in what has been called reality monitoring, the processes people use in deciding whether information contained in a memory had an internal or an external source.

Johnson and Raye (1981) proposed a model that attempts to explain our ability to make these discriminations, and to explain why reality monitoring sometimes fails. According to this model, information about the source of a memory is typically not directly encoded when the memory is formed; rather, identifying a memory's source is based on an evaluation of certain characteristics of the memory. These include the sensory and contextual attributes of the memory, the semantic detail

Preparation of this article was supported by NIMH Grant 5R01M4 3980902 to Ronald A. Finke, and by NSF Grant BNS-8510633 to Marcia K. Johnson. We thank Mary Potter for helpful comments on the project, and Laura Lekich for designing the forms. Requests for reprints should be addressed to Ronald A. Finke, Department of Psychology, State University of New York at Stony Brook, NY 11794, or to Marcia K. Johnson, Department of Psychology, Green Hall, Princeton University, Princeton, NJ 08544. contained in the memory, and the cognitive operations that were engaged when the memory was established. For instance, consider a memory for having recently seen an enjoyable movie. The memory would probably contain much more information about where and when the movie was seen, about the vividness of particular scenes, and about relationships among the characters, than would a memory based on merely having thought about seeing the movie. On the other hand, a memory based on pure fantasy would usually contain more information about the cognitive operations used to generate the memory. Thus, according to this view, specific characteristics of images, including how they were produced, determine whether they later can be discriminated from perception.

For example, one prediction of Johnson and Raye's model is based on the idea that mental images may include sensory qualities (e.g., see Finke \& Shepard, 1986; Kosslyn, 1980). According to the model, the more similar images are in sensory qualities to the particular perceived events from which they must be discriminated, the more difficult reality monitoring should be. In support of this prediction, Johnson, Raye, Wang, and Taylor (1979) found that, compared with poor imagers, vivid imagers were especially likely to overestimate how often they had seen pictures that they had both seen and imagined. Similarly, Anderson (1984) reported that memories for the imagined tracing of a pattern were more confusable with memories for actual tracings than were memories for looking at the pattern without imagining that it was 
being traced. Johnson, Foley, and Leach (in press) found that subjects had a more difficult time discriminating words they imagined from words they heard another person say if they imagined the words in the other person's voice rather than in their own. All of these findings indicate that similarity in sensory qualities between perceived and imagined events affects reality monitoring.

Apart from the sensory qualities of an image, how easily the image is generated is also predicted to affect reality monitoring. If the image is elicited with considerable reflection, effort, or elaboration, then the internally generated memory should be easier to distinguish from that for an external event than if the image is elicited naturally or spontaneously. Johnson, Kahan, and Raye (1984) found, for example, that people experience more confusion in reality monitoring for their actual dreams than for dreams they simply make up. Presumably, memories for real dreams contain less information about the cognitive operations used in the generation process, making them harder to distinguish from memories for perceived events.

There is also some evidence that memories based on normal, waking imagery can be confused with memories for perceived events, depending on how easily the images are generated. Durso and Johnson (1980) reported findings consistent with the idea that images generated as a spontaneous by-product of responding to questions about the referents of words (e.g., what is a knife used for?) are more likely later to be remembered as pictures than are images created purposefully in response to imagery instructions. Durso and Johnson, however, did not directly manipulate the ease of imaging in their study; thus, although their interpretation of their results was reasonable, it was also post hoc.

It should be possible to manipulate the ease of imagery experimentally. Studies on image generation have shown, for example, that it is easier to construct a mental image that corresponds to a familiar object or shape, or to a perceptually "good" figure (e.g., Kosslyn, Reiser, Farah, \& Fliegel, 1983; Murphy \& Hutchinson, 1982). We would predict, therefore, that any type of image that is easier than another to generate would lead to greater confusion in reality monitoring. Thus, one research strategy might be to vary the familiarity of the objects to be imagined. Evidence suggesting that this manipulation would affect reality monitoring comes from comparisons across experiments using different types of materials. For example, the overall level of reality monitoring accuracy was about $95 \%$ in a study in which subjects saw and imagined line drawings of common objects (Johnson, 1984) and only $56 \%$ in a study in which subjects saw and imagined letters of the alphabet (Kahan \& Johnson, 1984). Although there were many other differences between these studies, it is possible that the poor performance in the $\mathrm{Ka}$ han and Johnson study was a consequence of the greater ease with which subjects could generate vivid images of letters than of more complex objects.

In the present study, we adopted a different strategy to study the effect of ease of imagining on later reality monitoring. We explored memory confusions between observed and imagined completions of symmetrical visual patterns. There were two reasons for doing so. First, we suspected that how easily the imagined completions could be performed would depend on whether the forms were horizontally or vertically symmetrical. Previous studies have shown that judgments of symmetry can be performed most rapidly when using vertically symmetrical patterns (e.g., Corballis \& Roldan, 1975), suggesting that such patterns would be easier to imagine, and thus harder to distinguish in reality monitoring. This strategy allowed us to hold the materials constant (which is not possible with the strategy of manipulating preexperimental object familiarity) and to vary the ease of imagery simply by rotating the stimuli $90^{\circ}$. Second, by presenting half of a symmetrical pattern that is divided about the axis of symmetry, the imagined completion is specified without having to give detailed verbal instruction to the subjects.

\section{EXPERIMENT 1}

The first experiment was conducted to establish that halves of vertically symmetrical forms are regarded by subjects as easier to complete in imagination than halves of horizontally symmetrical forms. This finding is a prerequisite for conducting our second experiment, which explores memory confusions.

\section{Method}

Subjects. Twenty-four male and female undergraduate students at the State University of New York at Stony Brook served as volunteer subjects, receiving research credit in an introductory psychology course.

Stimuli. The stimuli consisted of 16 black and white outlined drawings of halves of symmetrical geometrical forms. They had the appearance of an incomplete figure that could be completed as a whole form if the missing half were added. The forms were chosen to be unfamiliar, yet distinguishable. Half of the forms could be made into vertically symmetrical figures, and half into horizontally symmetrical figures. They were divided equally on either side of the axis of symmetry; thus, there were 4 right halves, 4 left halves, 4 top halves, and 4 bottom halves. Examples of these patterns and their whole counterparts are shown in Figure 1.

Two groups of 16 stimuli were formed out of these patterns. The first group consisted of the 8 vertically symmetrical half forms and the 8 horizontally symmetrical half forms. The second group consisted of $90^{\circ}$ rotations of these same forms, so that the vertically symmetrical half forms in Group 1 became the horizontally symmetrical half forms in Group 2, and vice versa.

Procedure. The subjects were tested in small groups of 2 to 6 . Half of the subjects were shown the patterns for Group 1, and the other half the patterns for Group 2, which counterbalanced the axis of symmetry. Slides of the patterns were displayed in random order for 5 sec each using a Kodak Carousel projector, and were observed within a visual angle of $6.2^{\circ}$ at an average viewing distance of $2.2 \mathrm{~m}$. The subjects were instructed to imagine each half form as being completed about the axis of symmetry to make a symmetrical whole form. Once they had done so, they were to rate how easy or difficult it was to imagine completing the form, using a 5-point scale. These ratings ranged from a score of 1 (very easy) to a score of 5 (very difficult). After the slide was removed, the experimenter waited until all of the subjects had completed their ratings before presenting the 

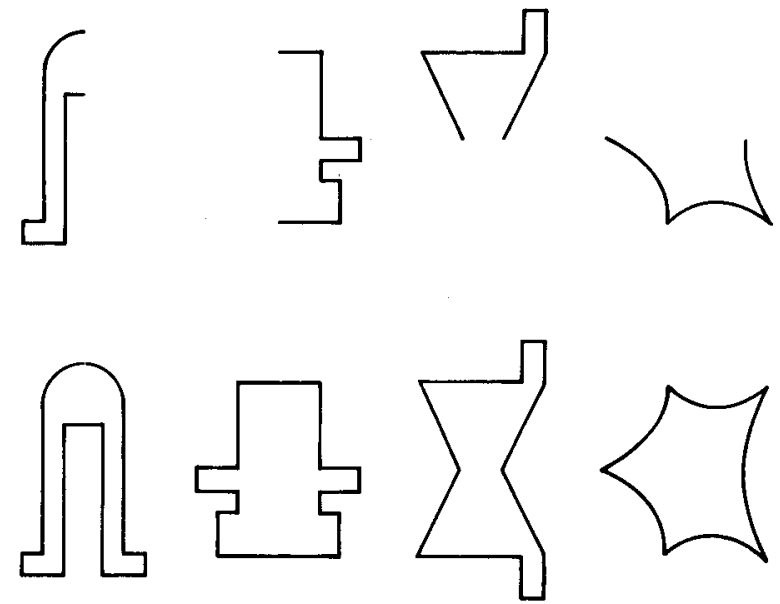

Figure 1. Examples of half and whole symmetrical forms used in Experiments 1 and 2.

next pattern. Before the experiment began, the subjects were shown examples of half forms and their whole-form counterparts, and were instructed about how the whole, symmetrical forms should look when completed.

\section{Results}

The mean rated difficulty of completing the forms was 1.86 for vertical stimuli and 2.50 for horizontal stimuli $[F(1,23)=24.80, p<.001]$. Thus, as predicted, subjects found it easier to imagine forms completed about the vertical axis of symmetry than about the horizontal axis.

\section{EXPERIMENT 2}

The results of the first experiment confirmed that horizontally symmetrical half forms are judged as being more difficult to imagine as complete than vertically symmetrical half forms. In Experiment 2, we tested our main prediction that the vertically symmetrical half forms would therefore produce greater confusion in reality monitoring when imagined as whole forms. That is, subjects should make more errors distinguishing between whether a form was imagined as complete or actually shown as complete when the forms are vertically symmetrical than when the forms are horizontally symmetrical.

\section{Method}

Subjects. A new group of 96 male and female subjects was selected as in Experiment 1.

Stimuli. The inspection stimuli consisted of two sets (A and B) of 16 forms each. Within each set, 8 were half forms that had been used in Experiment 1, and the other 8 were the whole versions of those forms. A third set of 8 whole forms, randomly selected from the original set of 24 forms, were used as distractor stimuli.

Procedure. As in Experiment 1, the subjects were tested in groups ranging from 2 to 6 . Half of the subjects were randomly assigned to an imagery condition, and half to a control condition. In both instruction conditions, the experiment began by presenting 16 of the inspection stimuli, in random order for $5 \mathrm{sec}$ each. For half the subjects, these consisted of the whole forms from Set A and the half forms from Set B; for the other half, they consisted of the whole forms from Set B and the half forms from Set A. Also, for half the subjects the forms were vertically symmetrical, and for the other half, horizontally symmetrical. Thus, whether for a particular form, its half or whole version was viewed and whether the form was vertically or horizontally symmetrical, were counterbalanced across subjects.

Subjects were told that if the slide contained a whole form, they were to rate how complex they thought the form was on a 5-point scale, where a rating of 1 meant very simple and a rating of 5 meant very complex. In the imagery condition, if the slide contained a half form, the subjects were to imagine it as a completed, symmetrical form (as subjects had done in Experiment 1), and then rate the complexity of the imagined whole form. In the control condition, if the slide contained a half form, the subjects were simply to rate the complexity of the half form itself, and no imagery instructions were given. Prior to the start of the experiment, the subjects were shown examples of whole and half forms; these example patterns were never included in the actual stimulus presentations.

The complexity rating procedure was a cover task used to make sure subjects attended to the forms and to decrease the possibility that they would attempt to prepare for a memory test. After completing their ratings, the subjects were shown 24 test patterns in random order. These consisted of the 8 whole forms they had just seen, 8 whole forms corresponding to completions of the half forms they had just seen, and 8 new whole forms. All forms were presented as vertically symmetrical for the vertical groups and horizontally symmetrical for the horizontal groups. Subjects were asked to indicate whether each pattern had been seen as a whole, contained half of a form they had seen, or had not been shown as a whole or as a half. It was explained to the imagery subjects that a form that was presented in half form but completed by imagination should be identified as a half. At the end of the experiment, the subjects were debriefed about the actual purpose of the study.

\section{Results}

Old-new recognition. First, the data were scored without regard to whether the subject remembered if the form had been presented as a half or a whole. These recognition scores were defined as the number of correct "old" responses (half items called half or whole plus whole items called half or whole) plus the number of correct rejections of new items, divided by the total number of test items (24). The percent correct recognition is shown in the first row of Table 1 . The main effect of instructions, the main effect of orientation, and the instructions $x$ orientation interaction were not significant (all $F_{\mathrm{s}}<1$ ).

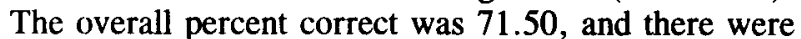
no differences among conditions. (An analysis of old-new recognition in terms of $d^{\prime}$ yielded similar results.)

The old-new recognition results enable us to make two points. First, comparison of the old-new recognition per-

Table 1

Results of Experiment 2

\begin{tabular}{lllll}
\hline & \multicolumn{4}{c}{ Experimental Conditions } \\
\cline { 2 - 5 } Response Measure & $\begin{array}{l}\text { Image Control } \\
\text { Vertical }\end{array}$ & $\begin{array}{c}\text { Image } \\
\text { Vertical }\end{array}$ Control \\
\hline $\begin{array}{l}\text { Percent Correct Recogntal Horizontal } \\
\text { (Hits + Correct Rejections) }\end{array}$ & .72 & .73 & .72 & .70 \\
$\begin{array}{l}\text { Half-Whole } \\
\text { Discrimination }\end{array}$ & .67 & .77 & .75 & .75 \\
\hline
\end{tabular}


formance of the two control groups indicates that the forms used in the experiment were equally memorable (in terms of recognition) when presented vertically and when presented horizontally. Second, the performance of the imagery and control groups on old-new recognition was comparable; hence, any differences between instructional groups in their ability to remember whether forms were presented as halves or wholes can be attributed to differences between groups in reality monitoring rather than in recognition.

Half-whole discrimination. A half-whole discrimination score was also computed for each subject: This score is analogous to the identification-of-origin score used in other reality monitoring studies (e.g., Johnson, Raye, Foley, \& Foley, 1981; Raye \& Johnson, 1980). In the present context, it was defined as the proportion of items identified as old that were also correctly identified as presented in half or whole form:

$$
[\mathrm{H}|\mathrm{H}+\mathrm{W}| \mathrm{W}] /[\mathrm{H}|\mathrm{H}+\mathrm{W}| \mathrm{H}+\mathrm{W}|\mathbf{W}+\mathbf{H}| \mathbf{W}] .
$$

A 2 (instructions) $\times 2$ (orientation) independent groups analysis of variance was conducted on these discrimination scores. There was a main effect of instructions $[F(1,188)=6.02, M S \mathrm{e}=.02, p<.01]$ and, more important, an instruction $\times$ orientation interaction $[F(1,188)$ $=4.81, M S \mathrm{e}=.02, p<.05]$. As can be seen in the second row of Table 1 , the imagery and control subjects did not differ in their ability to discriminate half from whole forms in the horizontal condition, whereas the performance of the imagery subjects was worse than that of the control subjects in the vertical condition.

Although we expected that the imagery subjects in the vertical condition would have more difficulty in reality monitoring than would the imagery subjects in the horizontal condition, we were initially surprised that the latter group showed no greater difficulty discriminating half from whole forms than did their controls. However, upon reflection, this is not strange. The half-whole discrimination of control subjects was only a little over $70 \%$, indicating that this was a relatively difficult discrimination, even in the absence of imagery instructions. The memory of engaging in effortful imagery (in the horizontal imagery condition) would provide cognitive operation cues that some forms were originally presented as halves. Furthermore, such cues would help offset confusion based on the sensory qualities of the completed images. Thus, whether an imagery group should show poorer reality monitoring relative to its no-imagery control depends on the balance in a particular task between costs from the image per se and benefits from the cues provided by memories for explicit imagery operations (see also Durso \& Johnson, 1980).

\section{GENERAL DISCUSSION}

To review, in Experiment 1, subjects viewed half a symmetrical form and were instructed to imagine the form in its complete version. Their ratings indicated that it was easier to imaginally complete forms symmetrical about the vertical axis than forms symmetrical about the horizontal axis. In Experiment 2, new subjects viewed these same forms presented either in their whole, symmetrical versions, or as half forms split along either the horizontal or vertical axis. Subjects in the imagery conditions were instructed to imaginally complete the half forms, whereas subjects in the control conditions did not receive imagery instructions. Compared with control subjects, imagery subjects had more difficulty remembering whether the form had been presented as a half or a whole in the case of imagined vertical completions. The imagined horizontal completions, which were presumably more difficult to contruct, did not produce a discrimination deficit.

The most straightforward interpretation of these results is that the greater difficulty that imagery subjects had in reality monitoring in the vertical than in the horizontal condition is attributable to the fact that images of vertically symmetrical forms are more easily constructed (as indicated by the results of Experiment 1); thus the memory for the construction includes less information about cognitive operations. Later, in evaluating the origin of a memory, low values for cognitive operations for imagined as well as perceived events would make it difficult to distinguish them (Johnson \& Raye, 1981). It is also possible that subjects in the vertical imagery condition not only could complete the forms more easily than could subjects in the horizontal imagery condition, but could construct better (i.e., more detailed) images as well. This would also reduce their ability later to discriminate half from whole forms. One interesting direction for further work would be to attempt to separately assess the individual contributions of ease of imagining and quality of the final image.

In many previous reality monitoring studies, particular events have been either wholly perceived or wholly imagined. One reason the present paradigm is interesting is that it approximates the common naturally occurring situation in which there is some perceptual support for an imagination. Our symmetrical form completion task is a visual analogue of studies of false recognition of inferences in prose comprehension. For example, a subject who has heard that a spy threw a secret document into the fireplace may later claim to have heard that the spy burned the document (e.g., Johnson, Bransford, \& Solomon, 1973). In such cases, the generated inference is not a complete fabrication, but rather an embellishment of presented information. Similarly, subjects imagining completed symmetrical forms are embellishing visually presented information (also see Jenkins, Wald, \& Pittenger, 1978). Compared with events that are entirely imagined, events that are partially perceived and partially generated should be particularly likely later to be misattributed to perception alone.

In summary, the present results are consistent with the idea that reality monitoring depends on the ease with which images are initially generated. The more effortlessly (or less reflectively) an image is generated, the 
harder it will later be to discriminate a memory of the image from memories of actual perceptual events.

\section{REFERENCES}

ANDERSON, R. E. (1984). Did I do it or did I only imagine doing it? Journal of Experimental Psychology: General, 113, 594-613.

Corballis, M. C., \& Roldan, C. E. (1975). Detection of symmetry as a function of angular orientation. Journal of Experimental Psychology: Human Perception \& Performance, 1, 221-230.

Durso, F. T., \& Johnson, M. K. (1980). The effects of orienting tasks on recognition, recall, and modality confusion of pictures and words. Journal of Verbal Learning \& Verbal Behavior, 19, 416-429.

Finke, R. A., \& ShEPARD, R. N. (1986). Visual functions of mental imagery. In K. R. Boff, L. Kaufman, \& J. P. Thomas (Eds.), Handbook of perception and human performance (Vol. 2). New York: Wiley-Interscience.

Jenkins, J. J., Wald, J., \& Pirtenger, J. B. (1978). In W. Savage (Ed.), Perception and cognition: Issues in the foundation of psychology. Minnesota studies in the philosophy of science (Vol. 9). Min neapolis: University of Minnesota Press.

JohnsON, M. K. (1984). [Memory for perceived and imagined pictures]. Unpublished raw data.

Johnson, M. K., Bransford, J. D., \& Solomon, S. K. (1973). Memory for tacit implications of sentences. Journal of Experimental Psychology, 98, 203-204.

Johnson, M. K., Foley, M. A., Leach, K. (in press). The consequences for memory of imagining in another person's voice. Memory \& Cognition.
Johnson, M. K., Kahan, T. L., \& RAyE, C. L. (1984). Dreams and reality monitoring. Joumal of Experimental Psychology: General, 113, 329-344.

Johnson, M. K., \& Raye, C. L. (1981). Reality monitoring. Psychological Review, 88, 67-85.

Johnson, M. K., Raye, C. L., Foley, H. J., \& Foley, M. A. (1981). Cognitive operations and decision bias in reality monitoring. American Journal of Psychology, 94, 37-64.

Johnson, M. K., Raye, C. L., Wang, A. Y., \& Taylor, T. H. (1979). Fact and fantasy: The roles of accuracy and variability in confusing imaginations with perceptual experiences. Journal of Experimental Psychology: Human Learning \& Memory, 5, 229-240.

Kahan, T. L., \& Johnson, M. K. (1984, April). Memory for seen and imagined rotations of alphanumeric characters. Paper presented at the meeting of the Eastern Psychological Association, Baltimore, MD.

KossLyn, S. M. (1980). Image and mind. Cambridge, MA: Harvard University Press.

Kosslyn, S. M., Reiser, B. J., Farah, M. J., \& Fuegel, S. L. (1983). Generating visual images: Units and relations. Joumal of Experimental Psychology: General, 112, 278-303.

Murphy, G. L., \& Hutchinson, J. W. (1982). Memory for forms: Common memory formats for verbal and visual stimulus presentations. Memory \& Cognition, 10, 54-61.

RAYE, C. L., JoHNSON, M. K. (1980). Reality monitoring vs. discriminating between external sources of memories. Bulletin of the Psychonomic Society, 15, 405-408.

(Manuscript received April 20, 1987; revision accepted for publication July 16,1987 .)

\section{Notice}

\section{Publications Office Accepts Manuscripts on Disk}

The Psychonomic Society Publications Office is now able to accept manuscripts on IBM PC or compatible floppy disks, and authors of accepted manuscripts are encouraged to make use of this new capability. The use of such disks, in conjunction with typewritten manuscripts, should both reduce journal production costs and shorten publication lag time.

To use this new system, send your disk containing the file to the journal's editor with the final typewritten revision of your accepted manuscript. Note that the electronic version should match exactly the hard-copy version of the manuscript.

Use an ASCII format on a 360-kilobyte, double-sided, double-density IBM PC or compatible disk, and be sure to indicate the name of the software used. Also, although not required, it would be helpful if the electronic version was adjusted to eliminate (1) hyphens at the ends of lines; (2) right-hand justifications; and (3) specialized word processor commands, such as those that create underlines, boldface, centering, tabs, and indents, or those that insert excessive space into the text, such as page commands. 